\title{
Estudio de un sistema de control interno del departamento de colecturía de la Unidad Educativa Particular Juan Montalvo del cantón la Concordia.
}

Study of an internal control system of the department of collector of the Particular Educational Unit Juan Montalvo of the canton Concord.

Sandra Karina Guamán Chávez, Ing. ${ }^{1}$, Ramiro Enrique Guamán Chávez, MSc. ${ }^{2}$, Jorge Luis Puyol Cortez, MSc., ${ }^{3} \&$ María Eulalia Alvear Lcda. ${ }^{4}$

DOI: https://doi.org/10.33262/visionariodigital.v2i1.37

\section{Resumen.}

Esta investigación se la realizo sobre el sistema de control interno en el departamento de colecturía de la Unidad Educativa Particular "Juan Montalvo" del Cantón La Concordia, esta área presenta deficiencia en el manejo y aplicación de normas de control interno porque existía mucha pérdidas económicas y materiales en la institución, en esta estudio se aplicó un metodología, crítica, prepositivo, descriptivo y exploratorio y se trabajó con un muestra de 247 personas divididas en 1 autoridad o rector de la institución, 9 docente, 3 personal administrativo y operativo, 114 padres de familia y 120 estudiantes y como resultado podemos indicar si socializamos las normas, políticas y manuales de procedimientos de control interno propuestos para el departamento de colecturía, se podrá controlar y verificar las debilidades de las diferentes áreas de un empresa o institución, esto conlleva también a un trabajo en conjunto y las autoridades del plantel cumplir para un sana convivencia en contexto educativo.

Palabras clave: Auditoría de gestión, Presupuesto educativo, Costes educativos, Administración de la educación.

\footnotetext{
${ }^{1}$ Unidad Educativa Particular Juan Montalvo, La Concordia, Ecuador sandritalvtlc@ hotmail.com

${ }^{2}$ Universidad Técnica Luis Vargas Torres de Esmeraldas, Extensión La Concordia, Ecuador, ramiro.guaman@utelvt.edu.ec

${ }^{3}$ Universidad Técnica Luis Vargas Torres de Esmeraldas Extensión La Concordia, Ecuador, jpuyol173@gmail.com

${ }^{4}$ Universidad Técnica Luis Vargas Torres de Esmeraldas Extensión La Concordia, Ecuador, marialvear@yahoo.com
} 


\begin{abstract}
.
This investigation was carried out on the internal control system in the collection department of the Particular Education Unit "Juan Montalvo" of Canton La Concordia, this area has a deficiency in the management and application of internal control standards because there was a lot of economic losses and materials in the institution, this study was applied a methodology, critical, prepositive, descriptive and exploratory and worked with a sample of 247 people divided into 1 authority or rector of the institution, 9 teacher, 3 administrative and operational staff, 114 parents of family and 120 students and as a result we can indicate if we socialize the rules, policies and manuals of internal control procedures proposed for the collection department, it will be possible to control and verify the weaknesses of the different areas of a company or institution, this also entails to work together and the campus authorities meet for a healthy coexistence in educational context.
\end{abstract}

Keywords: Management audit, Educational budget, Educational costs, Administration of education.

\title{
Introducción.
}

El Unidad Educativa Particular "Juan Montalvo", es una institución educativa de nivel secundario, que se encuentra ubicado en el Cantón de la Concordia. La institución educativa cuenta del departamento de Colecturía, la cual carece de Controles Internos que contribuya al adecuado funcionamiento y desarrollo de sus actividades. Es importante resaltar la trascendencia del departamento de Colecturía dentro de la institución educativa, ya que en ella se centra el manejo de los recursos económicos de la institución y de sus adecuado funcionamiento, de ello dependerá su desarrollo institucional, cabe mencionar que esta área se encuentra íntimamente relacionada con las áreas de bodega y suministros, es importante mencionar que es una institución de educación privada (Arimany, et. al. 2016).

En el recurrir del tiempo, el incremento poblacional, el desarrollo de las tecnologías, la importancia del departamento de colecturía no ha sido considerada en su totalidad, en virtud de que la mayoría de los establecimientos educativos no se han implementado normas de control interno que permitan regular el registro de sus actividades y operaciones con eficiencia, eficacia, y efectividad en beneficio de la institución (Ccaccya , 2015). Las constantes preocupaciones por reincidencias de fallas en los procesos, posibles fraudes o malos entendidos, el conformismo en obtener solamente lo suficiente para pagar sus facturas: no preocupándoles la dirección institucional ni el futuro de la entidad; a lo cual le agregaremos la falencia propia por falta de control interno operativo en la entidad, la probabilidad de que se incurran en pérdidas patrimonial será cada vez más acertada (Domínguez \& Benedicto, 2010). 
www.visionariodigital.org

Al evaluar en forma integral los procesos de control interno para luego tomar las medidas pertinentes que eviten incompatibilidades, conflictos de intereses, reserva de la información, y mitigar los riesgos de fraudes y errores operativos, financieros y legales (Ley Orgánica de Educación Intercultural , 2011). El Control Interno es sinónimo de evaluar la gestión y comprenderá un plan institucional basado en un conjunto de métodos y medidas adoptadas dentro de la entidad, a fin de salvaguardar sus recursos para verificar la exactitud y veracidad de su información financiera y administrativa, promover la eficiencia, eficacia y efectividad en las operaciones, estimulando la observación de las políticas prescritas e impulsar el cumplimiento de las metas y objetivos programados; ya que el control interno será una responsabilidad de todos de la institución (Jiménez , 2017).

Si una institución sea pública o privada descuidase de sus controles internos, su situación será tan frágil, que se podrá cometer fraudes, y errores que pondrán en riesgos el patrimonio. Un deficiente e inadecuado sistema de Control Interno en el departamento de colecturía del Unidad Educativa Particular "Juan Montalvo del cantón La Concordia, generara futuros problemas de tipo administrativo financiero dentro del funcionamiento institucional conllevando al retraso en el cobro de las mensualidades de los estudiantes, pago tardío de sueldos a la planta docente como administrativa ( López , 2013).

Es importante resaltar que el desarrollo de procedimientos que permitan optimizar el control y uso de los recursos, conllevara a la administración a tomar decisiones adecuadas que fomenten el desarrollo integral de la institución (MINEDUC, 2014), es por esto necesario que se tome la debida concientización ya que de no implementarse ese tipo de estrategias se vería afectada e involucrada en un riesgo y en un posible cese de sus actividades, por ende en un perjuicio para su población estudiantil ( Márquez, 2011).

El estudio de los sistemas de control interno tiene por objeto el avaluar si se salvaguarda o preserva los bienes de la entidad a fin de evitar desembolsos indebidos de fondos y si se ofrece seguridad de no contraer obligaciones sin autorización, la evaluación de los sistemas, procedimientos, reglamentaciones y actividades que interrelacionadas entre sí, la presente investigación tiende a realizar un estudio y evaluación e los sistemas y procedimientos de control interno a aplicarse en el departamento de colecturía de la institución (Quintero, 2009).

\section{Método y materiales.}

El tipo de investigación es de carácter de campo, con un con un método crítico, prepositivo, descriptivo y exploratorio, donde vamos observar las realidad de la problemática de la unidad educativa, además tiene un enfoque mixto cuantitativo cualitativo que permitirá recoger y analizar diferentes las razones del problema, se aplicara una encuesta a 247 personas divididas en 1 autoridad o rector de la institución, 9 docente, 3 personal administrativo y operativo, 114 padres de familia y 120 estudiantes. Para obtención de los resultados se utilizó como herramientas software Excel, formulas, 
www.visionariodigital.org

tablas estadísticas y para demostrar la magnitud del problema y poder proponer una propuesta se solución en este campo

Análisis de los datos.

Tabla 1. ¿Conoce usted las actividades y operaciones que realiza el departamento de Colecturía?

\begin{tabular}{|c|c|c|c|c|c|c|c|c|}
\hline \multirow[t]{2}{*}{ ALTERNATIVA } & \multicolumn{2}{|c|}{ AUTORIDAD } & \multicolumn{2}{|c|}{$\begin{array}{c}\text { PERS. } \\
\text { DOCENTE }\end{array}$} & \multicolumn{2}{|c|}{$\begin{array}{c}\text { PADRES DE } \\
\text { FAM. }\end{array}$} & \multicolumn{2}{|c|}{ ESTUDIANTES } \\
\hline & $\mathbf{F}$ & $\%$ & $\mathbf{F}$ & $\%$ & $\mathbf{F}$ & $\%$ & $\mathbf{F}$ & $\%$ \\
\hline SI & 0 & $0 \%$ & 2 & $40 \%$ & 10 & $14.08 \%$ & 20 & $27.03 \%$ \\
\hline $\mathrm{NO}$ & 1 & $100 \%$ & 3 & $60 \%$ & 0 & $85.92 \%$ & 54 & $72.97 \%$ \\
\hline TOTAL & 1 & $100 \%$ & 5 & $100 \%$ & 2 & $100 \%$ & 74 & $100 \%$ \\
\hline
\end{tabular}

Elaborado por: Grupo investigador.

De acuerdo a esta pregunta encontramos que, la autoridad principal de la institución desconoce las actividades y operaciones que debe realizar el área de colecturía ,el personal docente de plantel las conoce en un $40 \%$ de frente al $60 \%$ de desconocimiento, el personal administrativo y operativo asegura si conocerlo, el porcentaje de padres de familia que lo conocen es de $14.08 \%$ y el $85.92 \%$ no lo conocen y mientras que los estudiantes de la institución dicen conocerlas y el porcentaje equivale al $27.97 \%$. Dando a entender que dentro de la institución se está manejando de manera incorrecta la información debido a que la mayoría de los involucrados en ella desconocen las actividades y operaciones que debe realizar el área de colecturía.

Tabla 2. ¿Le interesaría a usted conocer las actividades que están bajo la responsabilidad del departamento de Colecturía?

\begin{tabular}{|c|c|c|c|c|c|c|c|c|c|c|}
\hline \multirow[t]{2}{*}{ ALTERNATIVA } & \multicolumn{2}{|c|}{ AUTORIDAD } & \multicolumn{2}{|c|}{$\begin{array}{c}\text { PERS. } \\
\text { DOCENTE }\end{array}$} & \multicolumn{2}{|c|}{$\begin{array}{l}\text { PERS. } \\
\text { ADMIN.Y } \\
\text { OPER. }\end{array}$} & \multicolumn{2}{|c|}{$\begin{array}{c}\text { PADRES } \\
\text { DE } \\
\text { FAM. }\end{array}$} & \multicolumn{2}{|c|}{ ESTUDIANTES } \\
\hline & $\mathbf{F}$ & $\%$ & $\mathbf{F}$ & $\%$ & $\mathbf{F}$ & $\%$ & $\mathbf{F}$ & $\%$ & $\mathbf{F}$ & $\%$ \\
\hline SI & 1 & $100 \%$ & 4 & $80 \%$ & 2 & $100 \%$ & 60 & $84.5 \%$ & 60 & $81.08 \%$ \\
\hline NO & 0 & $0 \%$ & 1 & $20 \%$ & 0 & $0 \%$ & 11 & $15.5 \%$ & 14 & $18.92 \%$ \\
\hline TOTAL & 1 & $100 \%$ & 5 & $100 \%$ & 2 & $100 \%$ & 71 & $100 \%$ & 74 & $100 \%$ \\
\hline
\end{tabular}

Elaborado por: Grupo investigador.

Respecto a la segunda pregunta encontramos que la autoridad el platel si está interesado a conocer las actividades y operaciones que se realiza el área de colecturía, el personal docente también se interesa por conocer en un $80 \%$ frente al $20 \%$ de negación, el personal administrativo y operativo, a pesar de conocer si desea aprender más sobre el tema los padres de familia asumen querer tener conocimiento en un $84.5 \%$ frente al $15.5 \%$ de 
www.visionariodigital.org

desinterés de los estudiantes del platel afirman querer conoce en un $81.08 \%$, mientras que el $18.92 \%$ no tienen interés en tener conocimiento sobre las actividades y operaciones que realiza el área de colecturía. Entiendo con los resultados que existe gran disposición en aprender comprender sobre las actividades y operaciones de área de colecturía para un correcto manejo y registro de las mismas.

Tabla 3. ¿Crees usted que el registro de las operaciones y actividades de colecturía se realizan de manera correcta?

\begin{tabular}{|c|c|c|c|c|c|c|c|c|c|c|}
\hline \multirow[t]{2}{*}{ ALTERNATIVA } & \multicolumn{2}{|c|}{ AUTORIDAD } & \multicolumn{2}{|c|}{$\begin{array}{c}\text { PERS. } \\
\text { DOCENTE }\end{array}$} & \multicolumn{2}{|c|}{$\begin{array}{c}\text { PERS. } \\
\text { ADMIN. } \\
\text { Y } \\
\text { OPER. }\end{array}$} & \multicolumn{2}{|c|}{$\begin{array}{c}\text { PADRES } \\
\text { DE } \\
\text { FAM. }\end{array}$} & \multicolumn{2}{|c|}{ ESTUDIANTES } \\
\hline & $\mathbf{F}$ & $\%$ & $\mathbf{F}$ & $\%$ & $\mathbf{F}$ & $\%$ & $\mathbf{F}$ & $\%$ & $\mathbf{F}$ & $\%$ \\
\hline SI & 1 & $100 \%$ & 0 & $0 \%$ & 1 & $50 \%$ & 8 & $11.27 \%$ & 48 & $64.86 \%$ \\
\hline $\mathrm{NO}$ & 0 & $0 \%$ & 5 & $100 \%$ & 1 & $5 \%$ & 63 & $88.73 \%$ & 26 & $35.14 \%$ \\
\hline TOTAL & 1 & $100 \%$ & 5 & $100 \%$ & 2 & $100 \%$ & 71 & 1005 & 74 & $100 \%$ \\
\hline
\end{tabular}

Elaborado por: Grupo investigador.

En la tercera pregunta se observa que, la autoridad del plantel está segura de las actividades y operaciones de colecturía si se están registrando de manera correcta a pesar de su ignorancia sobre el tema, por otro lado, el personal docente afirma que el área de colecturía no está cumpliendo correctamente con su trabajo, el personal administrativo y operativo lo afirma solamente en un 50\%, los padres de familia están conscientes que el área de colecturía no está trabajando correctamente en un $88.73 \%$ mientras el $11.27 \%$ asumen los contrario y en cuanto a los estudiantes del plantel el $64.86 \%$ dicen que si lo están realizando bien y el $35.14 \%$ lo niega. Dando a entender con esto que debería a desconocimiento sobre el tema algunos involucrados con la institución asumen que las actividades y operaciones de colecturía se están realizando de buen manera, mientras que los directamente afectados asegurando lo contrario.

Tabla 4. ¿Considera usted que se debería normar su actividad?

\begin{tabular}{|c|c|c|c|c|c|c|c|c|c|c|}
\hline \multirow[t]{2}{*}{ ALTERNATIVA } & \multicolumn{2}{|c|}{ AUTORIDAD } & \multicolumn{2}{|c|}{$\begin{array}{c}\text { PERS. } \\
\text { DOCENTE }\end{array}$} & \multicolumn{2}{|c|}{$\begin{array}{c}\text { PERS. } \\
\text { ADMIN. } \\
\text { Y } \\
\text { OPER. }\end{array}$} & \multicolumn{2}{|c|}{$\begin{array}{c}\text { PADRES } \\
\text { DE } \\
\text { FAM. }\end{array}$} & \multicolumn{2}{|c|}{ ESTUDIANTES } \\
\hline & $\mathbf{F}$ & $\%$ & $\mathbf{F}$ & $\%$ & $\mathbf{F}$ & $\%$ & $\mathbf{F}$ & $\%$ & $\mathbf{F}$ & $\%$ \\
\hline SI & 1 & $100 \%$ & 5 & $100 \%$ & 1 & $50 \%$ & 67 & $94.37 \%$ & 69 & $93.24 \%$ \\
\hline NO & 0 & $0 \%$ & 0 & $0 \%$ & 1 & $50 \%$ & 4 & $5.63 \%$ & 5 & $6.76 \%$ \\
\hline TOTAL & 1 & $100 \%$ & 5 & $100 \%$ & 2 & $100 \%$ & 71 & $100 \%$ & 74 & $100 \%$ \\
\hline
\end{tabular}

Elaborado por: Grupo investigador.

De acuerdo a la pregunta número cuatro encontramos que, la autoridad asegura que debería haber normativas para regular las actividades y operaciones del área de colecturía, el personal decente coincide con la aseveración del rector de la institución, 
www.visionariodigital.org

pero el personal administrativo solamente coincide en un 50\%, los padres de familia dicen en un $94.37 \%$ que si se debería norma su actividad, frente al $5.63 \%$ de negación y el porcentaje de los estudiantes de la institución que afirman sobre la normativa es del $6.76 \%$. Entendiendo con esto que se debe establecer las normativas para el área de colecturía.

Tabla 5. ¿Cree usted que los objetivos y metas institucionales se están logrando?

\begin{tabular}{ccccccc}
\hline ALTERNATIVA & AUTORIDAD & \multicolumn{2}{c}{$\begin{array}{c}\text { PERS. } \\
\text { DOCENTE }\end{array}$} & \multicolumn{2}{c}{$\begin{array}{c}\text { PERS. } \\
\text { ADMIN. Y } \\
\text { OPER. }\end{array}$} \\
\hline SI & $\mathbf{F}$ & $\mathbf{\%}$ & $\mathbf{F}$ & $\mathbf{\%}$ & $\mathbf{F}$ & $\%$ \\
NO & 0 & $0 \%$ & 1 & $20 \%$ & 0 & $0 \%$ \\
TOTAL & 1 & $100 \%$ & 4 & $80 \%$ & 2 & $100 \%$ \\
\hline
\end{tabular}

Elaborado por: Grupo investigador.

En la pregunta número cinco se obtiene que, la autoridad principal de la institución considere que no se están cumpliendo los objetivos y metas del plantel referente a ámbito económico, el personal docente del plantel coincide con el rector en un $80 \%$, mientras que el personal administrativo y operativo concuerda con la autoridad del plantel. Dando a entender con esto que las normativas y operaciones del área de colecturía son fundamentales para la consecución de los objetivos y metas institucionales.

Tabla 6. ¿Considera usted que se debe establecer sistemas de Control Interno en el departamento de Colecturía?

\begin{tabular}{|c|c|c|c|c|c|c|c|c|c|c|}
\hline \multirow[t]{2}{*}{ ALTERNATIVA } & \multicolumn{2}{|c|}{ AUTORIDAD } & \multicolumn{2}{|c|}{$\begin{array}{c}\text { PERS. } \\
\text { DOCENTE }\end{array}$} & \multicolumn{2}{|c|}{$\begin{array}{c}\text { PERS. } \\
\text { ADMIN. } \\
\text { Y } \\
\text { OPER. }\end{array}$} & \multicolumn{2}{|c|}{$\begin{array}{c}\text { PADRES } \\
\text { DE } \\
\text { FAM. }\end{array}$} & \multicolumn{2}{|c|}{ ESTUDIANTES } \\
\hline & $\mathbf{F}$ & $\%$ & $\mathbf{F}$ & $\%$ & $\mathbf{F}$ & $\%$ & $\mathbf{F}$ & $\%$ & $\mathbf{F}$ & $\%$ \\
\hline SI & 1 & $100 \%$ & 5 & $100 \%$ & 1 & $50 \%$ & 63 & $88.73 \%$ & 58 & $78.38 \%$ \\
\hline $\mathrm{NO}$ & 0 & $0 \%$ & 0 & $0 \%$ & 1 & $50 \%$ & 8 & $11.27 \%$ & 16 & $21.62 \%$ \\
\hline TOTAL & 1 & $100 \%$ & 5 & $100 \%$ & 2 & $100 \%$ & 71 & $100 \%$ & 74 & $100 \%$ \\
\hline
\end{tabular}

Elaborado por: Grupo investigador.

De acuerdo con la sexta pregunta encontramos que, que la autoridad del plantel si considera que se debe establecer el Control Interno en el área de colecturía, estando en concordancia con el personal docente y el $50 \%$ del personal administrativo y operativo de la institución, los padres de familia dicen estar de acuerdo en un $88.73 \%$, frente al $11.27 \%$ de negación, mientras que el $78.38 \%$ de los estudiantes afirman sobre el establecimiento de Control Interno. Obteniendo como respuesta que el Control Interno en el área de colecturía es esencial. 
Tabla 7. ¿Cree usted que las actividades y operaciones de colecturía mejorarían si se establece un adecuado sistema de control interno?

\begin{tabular}{|c|c|c|c|c|c|c|c|c|c|c|}
\hline \multirow[t]{2}{*}{ ALTERNATIVA } & \multicolumn{2}{|c|}{ AUTORIDAD } & \multicolumn{2}{|c|}{$\begin{array}{c}\text { PERS. } \\
\text { DOCENTE }\end{array}$} & \multicolumn{2}{|c|}{$\begin{array}{c}\text { PERS. } \\
\text { ADMIN. } \\
\text { Y } \\
\text { OPER. }\end{array}$} & \multicolumn{2}{|c|}{$\begin{array}{l}\text { PADRES } \\
\text { DE FAM }\end{array}$} & \multicolumn{2}{|c|}{ ESTUDIANTES } \\
\hline & $\mathbf{F}$ & $\%$ & $\mathbf{F}$ & $\%$ & $\mathbf{F}$ & $\%$ & $\mathbf{F}$ & $\%$ & $\mathbf{F}$ & $\%$ \\
\hline SI & 1 & $100 \%$ & 4 & $80 \%$ & 2 & $100 \%$ & 67 & $85.92 \%$ & 61 & $82.43 \%$ \\
\hline NO & 0 & $0 \%$ & 1 & $20 \%$ & 0 & $0 \%$ & 10 & $14.08 \%$ & 13 & $17.57 \%$ \\
\hline TOTAL & 1 & $100 \%$ & 5 & $100 \%$ & 2 & $100 \%$ & 71 & $100 \%$ & 74 & $100 \%$ \\
\hline
\end{tabular}

Elaborado por: Grupo investigador.

Referente a la pregunta número siete, la autoridad del plantel cree que las operaciones de colecturía mejorarían con el establecimiento de Control Interno, coincidiendo con el $80 \%$ del personal docente y con todo el personal administrativo y operativo, los padres de familia lo afirman en un $85.92 \%$ y los estudiantes del plantel lo aseguran en un $82.43 \%$. Entendiendo con esto que el Control Interno mejoraría el registro de las actividades y operaciones del área de colecturía.

Tabla 8. Si se implementa un sistema de Control Interno en el departamento de Colecturía ¿Usted cree que el ámbito financiero, administrativo y operativo del Colegio puede mejorar?

\begin{tabular}{|c|c|c|c|c|c|c|c|c|c|c|}
\hline \multirow[t]{2}{*}{ ALTERNATIVA } & \multicolumn{2}{|c|}{ AUTORIDAD } & \multicolumn{2}{|c|}{$\begin{array}{c}\text { PERS. } \\
\text { DOCENTE }\end{array}$} & \multicolumn{2}{|c|}{$\begin{array}{c}\text { PERS. } \\
\text { ADMIN. } \\
\text { Y } \\
\text { OPER. }\end{array}$} & \multicolumn{2}{|c|}{$\begin{array}{c}\text { PADRES DE } \\
\text { FAM. }\end{array}$} & \multicolumn{2}{|c|}{ ESTUIANTES } \\
\hline & $\mathbf{F}$ & $\%$ & $\mathbf{F}$ & $\%$ & F & $\%$ & $\mathbf{F}$ & $\%$ & $\mathbf{F}$ & $\%$ \\
\hline SI & 1 & $100 \%$ & 4 & $80 \%$ & 2 & $100 \%$ & 65 & $91.55 \%$ & 67 & $90.54 \%$ \\
\hline $\mathrm{NO}$ & 0 & 0 & 1 & $20 \%$ & 0 & $0 \%$ & 6 & $8.45 \%$ & 7 & $9.46 \%$ \\
\hline TOTAL & 1 & $100 \%$ & 5 & $100 \%$ & 2 & $100 \%$ & 71 & $100 \%$ & 74 & $100 \%$ \\
\hline
\end{tabular}

Elaborado por: Grupo investigador.

En la pregunta la autoridad del plantel afirma que la institución puede mejorar económicamente con el establecimiento de Control Interno, concuerda con su respuesta el personal administrativo y operativo, el $80 \%$ del personal docente, el $91.55 \%$ de los padres de familia y el $90.54 \%$ de los estudiantes del plantel. Obteniendo como resultado que si se pone en marcha el Control Interno en el área de colecturía la situación económica del Colegio sería mejor, contribuyendo en el engrandecimiento de la misma. 
wwW.visionariodigital.org

Tabla 9. ¿Usted considera que se debería realizar evaluaciones de cumplimiento a fin de determinar si las normas, procedimientos y políticas se cumplen?

\begin{tabular}{|c|c|c|c|c|c|c|c|c|c|c|}
\hline \multirow[t]{2}{*}{ ALTERNATIVA } & \multicolumn{2}{|c|}{ AUTORIDAD } & \multicolumn{2}{|c|}{$\begin{array}{c}\text { PERS. } \\
\text { DOCENTE }\end{array}$} & \multicolumn{2}{|c|}{$\begin{array}{c}\text { PERS. } \\
\text { ADMIN. } \\
\text { Y } \\
\text { OPER. }\end{array}$} & \multicolumn{2}{|c|}{$\begin{array}{l}\text { PADRES } \\
\text { DE } \\
\text { FAM. }\end{array}$} & \multicolumn{2}{|c|}{ ESTUDIANTE } \\
\hline & $\mathbf{F}$ & $\%$ & $\mathbf{F}$ & $\%$ & $\mathbf{F}$ & $\%$ & $\mathbf{F}$ & $\%$ & $\mathbf{F}$ & $\%$ \\
\hline SI & 1 & $100 \%$ & 5 & $100 \%$ & 1 & $50 \%$ & 55 & $77.46 \%$ & 41 & $55.41 \%$ \\
\hline NO & 0 & $0 \%$ & 0 & $0 \%$ & 1 & $50 \%$ & 16 & $22.54 \%$ & 33 & $44.59 \%$ \\
\hline TOTAL & 1 & $100 \%$ & 5 & $100 \%$ & 2 & $100 \%$ & 71 & $100 \%$ & 74 & $100 \%$ \\
\hline
\end{tabular}

Elaborado por: Grupo investigador.

En la novela pregunta la autoridad del plantel cree que se debe evaluar el control interno para saber que está siendo acatado correctamente, coincidiendo con el, el personal docente, el $50 \%$ del personal administrativo y operativo, el $71.46 \%$ de los padres de familia y el 55.41\%de los estudiantes del plantel. Dando entender con esto que el análisis es necesario para el Control Interno surja efecto positivo en el registro de las actividades y operaciones de la colecturía.

Tabla 10. ¿Usted estaría dispuesto a aplicar normas, manuales de procedimientos y políticas de control interno, determinado en el sistema propuesto?

\begin{tabular}{|c|c|c|c|c|c|c|c|c|}
\hline \multirow[t]{2}{*}{ ALTERNATIVA } & \multicolumn{2}{|c|}{ AUTORIDAD } & \multicolumn{2}{|c|}{$\begin{array}{c}\text { PERS. } \\
\text { DOCENTE }\end{array}$} & \multicolumn{2}{|c|}{$\begin{array}{c}\text { PERS. } \\
\text { ADMIN. Y } \\
\text { OPER. }\end{array}$} & \multicolumn{2}{|c|}{$\begin{array}{c}\text { PADRES DE } \\
\text { FAM. }\end{array}$} \\
\hline & $\mathbf{F}$ & $\%$ & $\mathbf{F}$ & $\%$ & $\mathbf{F}$ & $\%$ & $\mathbf{F}$ & $\%$ \\
\hline SI & 1 & $100 \%$ & 5 & $100 \%$ & 2 & $100 \%$ & 63 & $88.73 \%$ \\
\hline NO & 0 & $0 \%$ & 0 & $0 \%$ & 0 & $0 \%$ & 8 & $11.27 \%$ \\
\hline TOTAL & 1 & $100 \%$ & 5 & $100 \%$ & 2 & $100 \%$ & 71 & $100 \%$ \\
\hline
\end{tabular}

Elaborado por: Grupo investigador.

La autoridad de la intuición educativa si estarían de acuerdo en cumplir las normativas, acordado conjuntamente con el personal docente, administrativo y operativo y los padres de familia en un $88.73 \%$, mientras $11.27 \%$ no estaría de acuerdo con este o desconoce. Entendiendo con esto que los individuos inmersos con la institución están pestos para ayudar a mejorar la situación del Colegio Técnico Particular Mixto Popular "Juan Montalvo", del cantón la Concordia.

\section{Conclusiones.}

- Proponer el establecimiento de un sistema de Control Interno en el departamento de Colecturía de la Unidad Educativa Particular "Juan Montalvo, del cantón La 
Concordia, a todos los individuos relacionados con la institución, esto alcanzara buenos beneficios al aplicar y cumplir normativa para el mejoramiento de las actividades y control de recursos económico en para la mejora de la institución.

- La inclusión de los directivos y personal administrativos estudiante y padres de familia sobre de los manejos de políticas institucionales con es el caso de manejo y las normas del control interno con el fin de poder solucionar la problemática y mejorara la imagen de la institución.

- Socializar las normas, políticas y manuales de procedimientos de control interno propuestos para el departamento de colecturía, con esto se podrá controlar y verificar las debilidades de las diferentes áreas de una empresa o institución, además las autoridades del plantel deben buscar estrategias para cumplir las actividades de acuerdo a las normativas y políticas de los procedimientos establecidos con el objeto de promover un desarrollo sustentable en la institución.

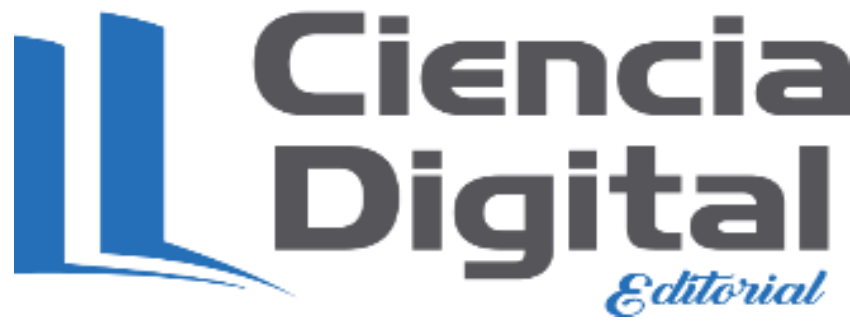




\section{Para citar el artículo indexado.}

Guamán S., Ing. , Guamán R., Puyol J., \& Alvear M.., (2018). Estudio de un sistema de control interno del departamento de colecturía de la Unidad Educativa Particular Juan Montalvo del cantón la Concordia. Revista electrónica Visionario Digital1(1), 65-75. Recuperado desde:

http://cienciadigital.org/revistacienciadigital2/index.php/VisionarioDigital/article/view/37/ $\underline{36}$

\section{【Liencia}

El artículo que se publica es de exclusiva responsabilidad de los autores y no necesariamente reflejan el pensamiento de la Revista Ciencia Digital.

El articulo queda en propiedad de la revista y, por tanto, su publicación parcial y/o total en otro medio tiene que ser autorizado por el director de la Revista Ciencia 